\title{
Advances in experimental assessment of dynamic tensile strength of concrete by the spalling technique
}

\author{
... in tribute to Janusz Roman Klepaczko, Emeritus Professor at the Université Paul Verlaine in Metz, passed away on August 15, 2008.
}

\author{
Ahmed Brara ${ }^{\mathrm{a}}$ \\ National Center of Studies and Integrated Research on Building Engineering, Cité El Mokrani, Algiers, Algeria
}

\begin{abstract}
An experimental method to test concrete in dynamic tension by spalling with a Hopkinson bar as loading and measuring tool was developed in 1999. The dynamic strength of concrete specimen and strain rate were indirectly derived from an accurate data processing of the signals measured on the Hopkinson bar surface. This method suggested by late Prof. Klepaczko, allowed for reaching the highest strain rate reported in literature for which an intriguing tensile strength increase was highlighted. This simple and efficient technique has been adopted by many researchers around the world. Some significant improvements in terms of definition and reproducibility of the incident loading pulse travelling along the bar and direct and/or contactless measurements on concrete specimens have been introduced. The very high rate sensitivity of concrete tensile strength was corroborated by the additional experimental data obtained with this experimental technique during the last fifteen years.
\end{abstract}

\section{Introduction}

The most important phenomenon highlighted by the experimentation on concrete dynamics undertaken during the last decades is the rate sensitivity of the material, which means that its mechanical properties - notably the tensile strength, the Young's modulus, the Poisson ratio, the critical strain - depend on the applied loading rate. The phenomenon of increase of tensile strength with the loading rate, observed in the early sixties of the last century has been recognized as the "rate effect", and is typically represented as a dynamic increase factor (DIF), i.e., the ratio of dynamic to quasi-static strength versus strain rates. Globally, the rate dependency of concrete tensile strength can be subdivided into two regimes; a regime starting from the quasi-static range of strain rates up to about $1.0 \mathrm{~s}^{-1}$ with moderate rate effects and a regime with extensive rate effects for higher strain rates for which a DIF increased from 2 to about 7 [1]. The highest strain rates were essentially obtained with techniques based on the spalling phenomenon. Spall is defined as the ejection of fragments of a material specimen from the opposite side from which the specimen was impacted and/or impulsively loaded.

The earliest experiment at very high strain rate reported by the literature was conducted on large plain concrete specimens (51 $\mathrm{mm}$ diameter and $838 \mathrm{~mm}$ length) impacted at one end by a steel sphere. The signals were recorded by means of strain gauges cemented on concrete specimen surface. In these tests, DIFs of 2.5 and 6 for $2.0 \mathrm{~s}^{-1}$ and $23 \mathrm{~s}^{-1}$ were respectively highlighted [2].

This considerable strength increase was also observed about two decades later for large concrete instrumented wall panels $(4,57 \mathrm{~m} \times 1,65 \mathrm{~m})$, each which were mounted on the side of a rectangular box reaction structure, loaded

\footnotetext{
${ }^{a}$ Corresponding author: ahmedbrara@hotmail.com
}

by surface explosion of TNT charge [3]. Pullback velocity of the ejected fragments measured during the test by means of high speed photography $\left(10^{3}\right.$ pictures/s) allowed estimating the spall strength whereas strain rates were assessed from the strain gauges measurements cemented in the vicinity of the explosion zone of the concrete panel. For an estimated strain rate of $157 \mathrm{~s}^{-1}$ a $\mathrm{DIF}=7$ was determined. A tenfold increase of tensile strength was also previously mentioned in 1978 for concrete structures impacted by missiles [4].

It should be noted that up to the mid 90ties, the experimental data concerning the dynamic tensile behavior of concrete were rather scarce or inexistent, notably at strain rates higher than $20 \mathrm{~s}^{-1}$. This crucial need of additional experimental data on dynamic concrete tensile behaviour led to the development in 1999 of a new experimental arrangement in the Laboratory of Physics and Mechanics of Materials (LPMM) of Metz University (France). The idea inferred from an intensive study of the existing testing methods [5], was to combine the old spall technique for reaching high strain rate combined with a Hopkinson bar as reliable measuring tool by introducing the recent developments achieved in signals processing. This testing configuration was already adopted for characterizing the dynamic tensile behavior of ceramics in the 1D framework, amongst others [6].

One of the most difficult problems encountered for such kind of design, was to find an optimal compromise between the antagonistic effects induced by the dimensions (diameters $\phi$ and lengths $L$ of striker, bar and specimen) of rods when used as mechanical waveguides. Indeed, the specimen size must be from one side sufficiently large and representative of the concrete material with avoiding scale effects due to its heterogeneity (aggregates essentially), from another side shortest striker lengths 


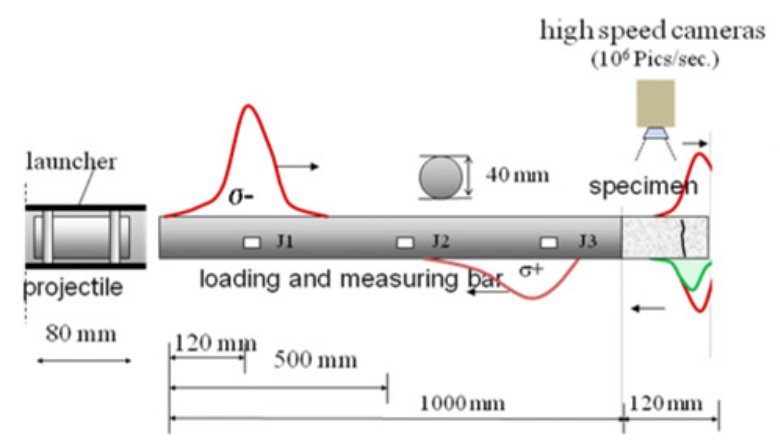

Figure 1. Scheme of experimental setup.

must be adopted in order to reach very high strain rates. However, the dynamic testing on rods (specimens and/or Hopkinson) with large diameters and short lengths $\left(\mathrm{L}_{\mathrm{p}}\right)$ of striker (and then short wavelength $\lambda$ ) can introduce notable oscillations (radial and longitudinal dispersion of the propagating waves) which can spoil the measurements, and leads also to mechanical difficulties in controlling testing devices. It is known since the Davies work [7] that rod with finite lengths and short wavelengths induce in any case geometrical dispersion. From the cumulated experimental knowledge, it is generally admitted, for assuming reasonably an uniaxial longitudinal wave propagation in elastic bars, empirical conditions concerning their dimensions (length $\mathrm{L}$ and diameter $\phi$ ) and wavelength $\lambda$ must be satisfied, for instance [8]. Thus, the rod length-to-diameter and wavelength-to-rod ratios must respectively verify $\mathrm{L} / \phi>20$ for an uniaxial and homogeneous deformation along the cross section of the rod and $\lambda / \phi \sim 2 \mathrm{~L}_{\mathrm{p}} / \phi>5$ for a propagation with a constant velocity $\mathrm{C}_{0}=(E / \rho)^{1 / 2}$ (with $\rho$ being the mass density of the rod).

In this paper, the evolution of this method and some major modifications introduced during the last years are presented and discussed. Because of the numerous adaptations, only some representative configurations will be evoked in this paper.

\section{Experimental methods based on spalling technique}

\subsection{The original setup developed in LPPM of Metz university}

This experimental arrangement consisted of an air launcher of cylindrical projectile with a Hopkinson bar as a measuring tool and a cylindrical concrete specimen in contact with the bar, see the scheme on Fig. 1. The projectile, the Hopkinson bar made of the same aluminium alloy and the concrete specimens have the same diameter $(40 \mathrm{~mm})$ with respective length of $80 \mathrm{~mm}$, $1000 \mathrm{~mm}$ and $120 \mathrm{~mm}$. The incident compressive wave transmitted by the Hopkinson bar into the specimen is reflected as tensile wave causing spalling. The incident and reflected waves produced after striker impact are measured by three SR gage stations of high sensitivity cemented on the Hopkinson bar surface. The experimental technique is on the other hand combined with "up-todate" instrumentation (adapted to dynamics strain gauges, high rate sampling oscilloscopes ...) and equipment like high-speed video-cameras $\left(10^{6}\right.$ pictures/sec.). The values of the chosen ratios Hopkinson bar diameter to its length $\mathrm{L}_{b} / \phi_{\mathrm{b}}=25$ and wavelength to bar diameter $\lambda / \phi_{\mathrm{b}} \sim 5$ correspond in fact to the lower limits for assumption of an unidimensional analysis of longitudinal wave propagation.

A special numerical procedure has been applied for analyses of wave process measured by the SR stations on the Hopkinson bar. The main task was the exact reconstitution of the transmitted wave $\sigma_{t}$ into specimen via the interface bar-specimen by using the incident compression wave together with the reflected one in the Hopkinson bar. The signals are shifted by calculating phase velocity of their frequency components by the accurate resolution of the known Pochammer [9] Chree [10] "frequency equation" and the correction of their geometrical dispersion of using Fourier analysis. The dynamic phase velocity in the Hopkinson bar was determined by the superposition method.

For same reasons (that is, avoiding of the effect of dispersive phenomena due to specimen dimensions and short wavelenght), indirect assessment of failure strength and loading rate by numerical simulation of the propagation of the transmitted pulse $\sigma_{t}$ and deduction of spall strength $\sigma_{f}$ and loading rate corresponding to the failure position $\mathrm{Z}_{c}$, in an assumed framework of $1 \mathrm{D}$ analysis. The rate sensitivity of the Young's modulus $\mathrm{E}_{\text {dyn }}$ was calculated according to the relation already established by Toutlemonde [11],

$$
E_{d y n}=E_{0}+\alpha \phi(H) \log \left(\dot{\sigma} / \dot{\sigma}_{0}\right)
$$

where $E_{0}$ and $\dot{\sigma}_{0}$ are de quasi-static Young's modulus and loading rate respectively and $\alpha, \phi(\mathrm{H})$ materials characteristics. More details can be found in [12] or in [13].

A large experimental program was conducted on wet and dry micro-concrete cylindrical specimens. The tested concrete was so called "micro-concrete" MB50, because of small size of the aggregates used (maximum size: $2 \mathrm{~mm}$ ). Many experiments have been performed on this reference material within two successive research programs of the networks GEO and GEOMATERIAUX, carried out by several French laboratories $[14,15]$. These important research programs conducted during the $90^{\text {ties }}$ were devoted to the general topic of "the behaviour of geomaterials and structures in their environment", including "behaviour of concrete in high rate dynamics" in which late Professor Janusz R. Klepaczko and his numerous co-workers in LPMM have taken an active part.

Several series of spall tests on micro-concrete specimens of diameter $40 \mathrm{~mm}$ and $120 \mathrm{~mm}$ length were performed with different impact velocities of striker. Depending on the impact velocity, one, two and even three flat failures were obtained. For the entire sample of specimens tested, no simultaneous fractures were observed. Moreover, the post mortem analysis of the broken specimens revealed only a single fracture plane, and no diffuse microfissuration was detected in its vicinity. For the data assessment, only the first fracture was taken into account, because the fracture process of specimen generates high frequency oscillations influencing strongly the residual tensile stress as experimentally observed by 


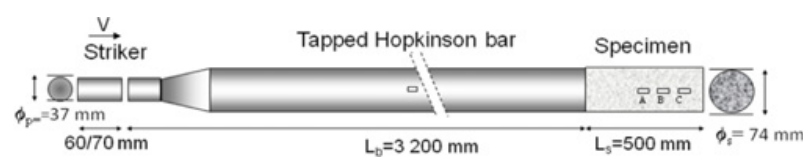

Figure 2. Experimental arrangement with tapped incident bar.

Bierwirth [6] for ceramics and clearly established later by Galvez Diaz-Rubio [16], and Gomez del Rio [17]. The chronology of the fracture sequences were clearly established with the CCD captured pictures.

The experimental program by application of this technique at strain rates ranging between $20 \mathrm{~s}^{-1}$ and $120 \mathrm{~s}^{-1}$ has permitted to confirm very precisely the substantial increase of the tensile strength mentioned earlier by Birkimer [1] and Mac Vay [2]. Moreover, this increase was found without apparent limit, since at strain rates of about $120 \mathrm{~s}^{-1}$, the dynamic tensile strength rises to 10 times the quasi-static strength! This strength enhancement was corroborated independently by the analysis of pictures obtained for every test by the fast video cameras. Series of captured pictures for each test during the failure process enabled to estimate the dynamic tensile strength through the fragment velocity (linearly related to the concrete impedance in 1D longitudinal uniaxial wave propagation approach) [12]. Numerical simulations of the tests with Discrete and Finite Elements Methods assuming an elastic brittle behaviour of concrete and a fracture plane were in accordance with the experimental results [13]. This confirmation via non contact measurements (video cameras) and the numerical simulations validates the reasonable assumption of an uniaxial wave propagation in the concrete specimen and the elastic-brittle behaviour of the material.

This efficient configuration to test concrete via spalling was adopted worldwide by numerous researchers with introducing some major modifications in the measurements and/or the compressive pulse generating methods, as chronologically (but non-exhaustively) reported below.

\subsection{Advances in the experimental technique}

The same arrangement was adopted by Wu et al. [18] consisting of a cylindrical projectile $(70 \mathrm{~mm}$ length, $\phi$ $37 \mathrm{~mm}$ ) impacting a partially taped incident pressure bar of $3200 \mathrm{~mm}$ length cemented to a $500 \mathrm{~mm}$ long cylindrical concrete specimen, Fig. 2.

The projectile length and dimensions of the taped section of the incident pressure bar were designed in order to ensure a transmitted stress wave in the concrete specimen in a repeatable form of an almost half periodic sine shaped wave of about $\sim 100 \mu$ s duration. The concrete specimen and the incident pressure bar have the same diameter $(74 \mathrm{~mm})$. With the strain-time signal recorded by strain gauges glued on the concrete specimen, the dynamic tensile stress was calculated according the one-dimensional stress wave theory for viscoelastic material (however only the stress wave attenuation was taken into account in experimental data analyses, the geometrical dispersion was considered as not significant). The spall strength and strain rate at crack location were obtained by numerical simulation of the transmitted

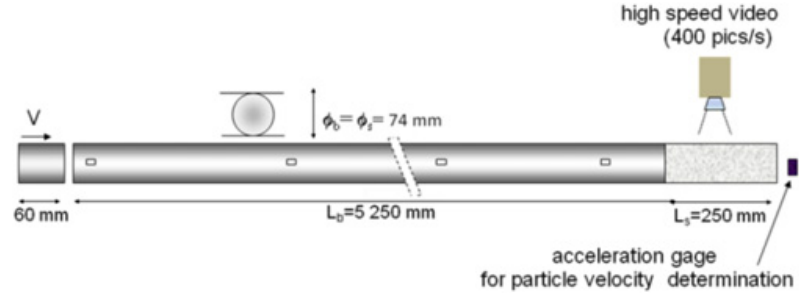

Figure 3. Experimental device with accelerometer for spall strength measurement.

compressive pulse superposition process. For the current concrete (with a compressive strength of $34 \mathrm{MPa}$ ) tested, similar results as those obtained in LPMM were reported. At strain rates ranged from $20 \mathrm{~s}^{-1}$ to about $90 \mathrm{~s}^{-1}$, the DIF was found to vary from 2 to 9 .

This experimental arrangement with partially tapped bar was also used to study the dynamic behavior of ultrahigh performance cement based composites (UHPCCs) intended to defense works, with $200 \mathrm{MPa}$ compressive strength [19]. The composites were prepared with natural sand and by replacing a large quantity of cement by industrial waste residues such as silica fume, fly ash and slag, and different fiber volume fractions. For these UHPCCs, DIF of 1.6 to 6.7 were found for strain rate increasing from $21 \mathrm{~s}^{-1}$ to $66 \mathrm{~s}^{-1}$.

To study tensile strength and fracture energy of current concrete (maximum aggregate size of $8 \mathrm{~mm}$, compressive strength of $40 \mathrm{MPa}$ ), Schüler et al. (2006) [20] used a incident bar and notched and unnotched concrete specimens of $74.2 \mathrm{~mm}$ diameter with respective length of $\mathrm{L}_{\mathrm{b}}=5500 \mathrm{~mm}$ and $\mathrm{L}_{\mathrm{s}}=250 \mathrm{~mm}$, Fig. 3. In order to measure the waves, four strain gage stations were applied at the Hopkinson bar surface, whereas the free surface velocity at the end of the specimen was determined via integration of the acceleration signal obtained with a piezoresistive accelerometer. The setup was also equipped with fast video cameras (400 pictures/sec.) in order to capture the fracture sequences.

For the data processing, almost the same procedure as in the LPMM experiment was adopted where the recorded compressive stress pulse is shifted at the bar end by taking into account the geometrical dispersion.

The wave velocity $\mathrm{C}_{0}=\mathrm{L}_{\mathrm{s}} / \Delta \mathrm{t}$ and therefore the dynamic Young's modulus $\mathrm{E}_{\mathrm{dyn}}=\rho \mathrm{C}_{0}^{2}$ of the tested concrete were calculated knowing the time $\Delta \mathrm{t}$ needed by the wave to propagate along the specimen. Having the measured free surface velocity $\Delta \mathrm{V}_{\mathrm{pb}}$ at the end of the specimen, the spall strength was derived as done for plate impact experiment for 1D strain conditions [21], that is

$$
\sigma_{f}=1 / 2 \rho C_{0} \Delta V_{p b} .
$$

At strain rates ranging between $31 \mathrm{~s}^{-1}$ to $68 \mathrm{~s}^{-1}$ a DIF of 4 to 5.5 for current concrete (with $8 \mathrm{~mm}$ maximum aggregate size) was reported.

By means of this setup developed by Schuler et al. [20], dynamic spall experiments at strain rates varying between 140 and $180 \mathrm{~s}^{-1}$ were conducted on notched and unnotched cylindrical specimens of strain hardening cement-based composites (SHCC) [22]. The SHCCs are characterized by 


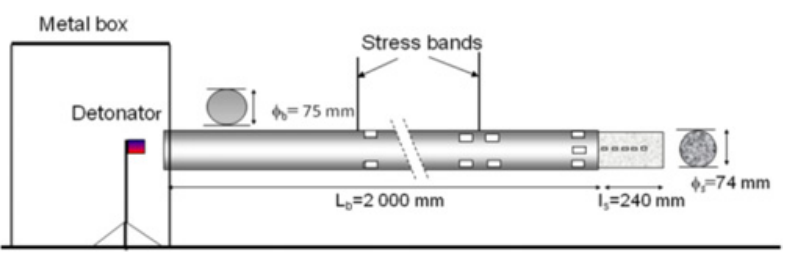

Figure 4. Setup with compressive wave generated by small explosive charge.

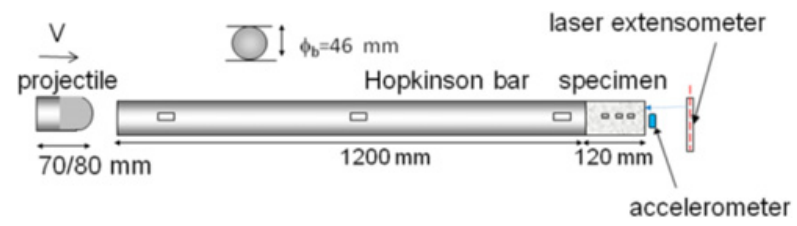

Figure 5. Spalling test with (and non-)contact measurements.

very high binder and relatively high fiber volume contents in finely grained matrix composition $(0.2 \mathrm{~mm}$ maximum aggregates size) with fly ash. At strain rate of about $\sim 140 \mathrm{~s}^{-1}$, a DIF of 6.7 was highlighted for this material.

For the similar research aim as the precedent author, a setup based on the same principles was developed by Weerheijm et al. [23] consisting of an horizontal steel bar (length $2000 \mathrm{~mm}, \emptyset 75 \mathrm{~mm}$ ), supported by strings, at the end of which a concrete specimen of $240 \mathrm{~mm}$ length and $74 \mathrm{~mm}$ diameter was attached, Fig. 4.

The short compressive shock wave $(\sim 70 \mu \mathrm{s})$ was introduced into the rod by detonating a small explosive charge at other end of the bar. The loading rate and applied load were derived from the strain measurements on the steel bar and the specimen. The transmitted pulse was determined via the incident compressive pulse recorded with carbon gauges embedded in the concrete specimen and the dynamic Young modulus $\mathrm{E}_{\mathrm{dyn}}$ (deduced from the wave velocity $\mathrm{C}_{0}$ as done by Schuler et al. [20]), and calculated using the transmission time between the different strain gages locations distributed near the contact interface with the bar and along the rear half of the specimen intended to capture the failure zone. Assuming an uniaxial wave propagation, the transmitted tensile pulse was reconstructed theoretically, whereas the dynamic tensile strength was deduced from strain measurements just outside the analytically (and via exploring tests) predetermined failure zone. The geometrical dispersion was not taken into account in data processing.

A set of notched and un-notched concrete specimen were tested. The material studied was a current concrete with $8 \mathrm{~mm}$ maximum aggregate size and a compressive strength of $40 \mathrm{MPa}$. For the unnotched concrete specimen a DIF of 5.3 for strain rate of $20 \mathrm{~s}^{-1}$ was found.

An experimental device that possesses almost the same features of the previous setup developed in the LPMM was rebuilt by Erzar and Forquin [24]. The dimensions of the Hopkinson and striking bars made of aluminum alloy and concrete specimens were nearly the same as in the original setup (projectiles with $46 \mathrm{~mm}$ diameter and $70 / 80 \mathrm{~mm}$ length, bar and specimens length of $1200 \mathrm{~mm}$ and $120 / 140 \mathrm{~mm}$ respectively with same diameter of $45 \mathrm{~mm}$ ), Fig. 5. Some modifications were introduced like

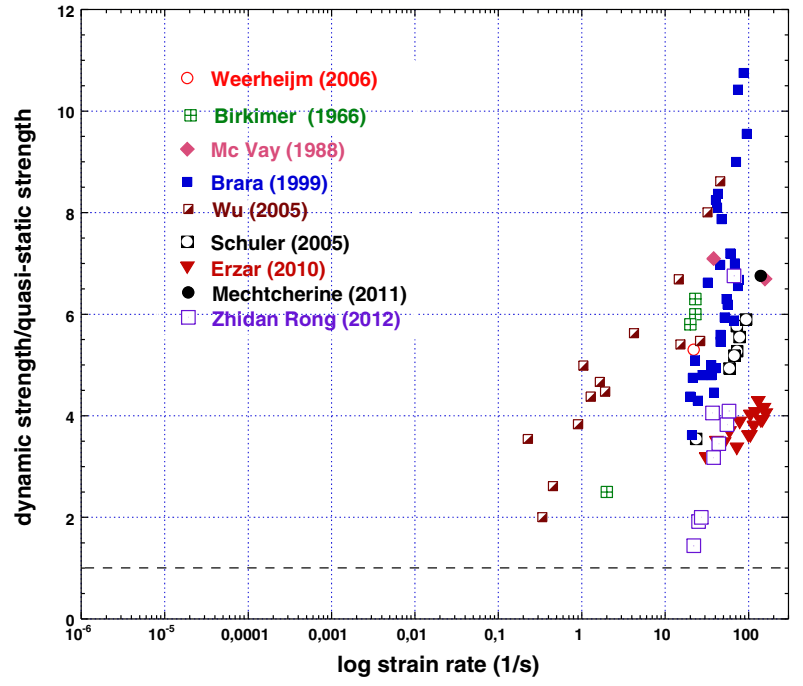

Figure 6. Dynamic increase factor as function of strain rate (log, $1 / \mathrm{s})$.

the use of hemispheric smooth-end projectiles in order to improve the homogeneity of tensile loading in the specimen and metrology instruments such as piezoresitive accelerometer and laser extensometers.

To measure particles velocities (and further the spall strength), laser extensometers were placed on the free end surface specimen. The wave speed $\mathrm{C}_{0}$ was derived from the difference between the time corresponding to the minimum value of strain of the first gauge placed on the specimen near the interface and the time corresponding to the maximum value of velocity measured by the laser extensometer. Furthermore, the dynamic Young's modulus estimated from $\mathrm{C}_{0}$ allowed for deducing the spall strength $\sigma_{f}$, assuming an uniaxial longitudinal wave propagation. The dynamic strength being known, the gauge signal was used to identify accurately the "failure time". The change of the strain-rate in the damaged area was time-derived from gauge signals and the strain at failure time was finally deduced. The position of failure plan responsible of velocity rebound recorded by the extensometer on the rear face of the specimen was precisely identified, starting from travel time of waves along the bar. This position permitted to locate the strain gauge for strain rate characterizing. In this investigation, the wave dispersion effects induced by the short loading pulse $(\sim 70 \mu \mathrm{s})$ were neglected.

For the several tests performed on a set of cylindrical MB50 micro-concrete (already studied in LPMM) specimens, relatively low values of DIF were found for strain rate varying from 3 to about 5 at strain rates ranged from $30 \mathrm{~s}^{-1}$ to $150 \mathrm{~s}^{-1}$.

Experimental results of the last decades are gathered on Fig. 6, where the tensile DIF versus the logarithm of strain rate is plotted. The studied material was generally dry and wet micro-, mini- or current and fiber reinforced concrete (from $2 \mathrm{~mm}$ to $25 \mathrm{~mm}$ maximum aggregate sizes), with different compressive strengths. Note that, normalizing data with respect to the quasi-static values may eliminate problems such as different maturities (relative to curing time), different mix strengths and eventual scale effects due to both specimen and aggregates sizes. 
As illustrated by the Fig. 6, practically all the data obtained with the experimentation based on spalling technique show the same trend and corroborate the steep increase of the relative tensile strength at high strain rate.

\subsection{Discussion}

The retrospective of the development in recent years of experimentation on concrete based on the technique of spalling highlighted significant advances. Beside a better reproducibility of loading signals (via pulses shaping or loading with explosive charges), direct measurements on concrete specimens were introduced. Means of measurements with (or without) contact have been added such as accelerometers and laser extensometers next to those used previously, namely the strain gauges and fast video cameras but with largely improved performances. The accelerometer is a miniature device encapsulated in a small lightweight aluminum alloy box (less than $10 \mathrm{~mm}^{3}$ in volume and weighing approximately $2.5 \mathrm{~g}$ ) allowed to measure peak accelerations of $600000 \mathrm{~m} / \mathrm{s}^{2}$. Glued on the rear face of specimen, it allows, as in Schuler et al. [20] and Weerheijm et al. [23] tests, to measure the pullback velocity and next to calculate the dynamic resistance of the specimen knowing the wave velocity $\mathrm{C}_{0}$ and the Young's modulus $\mathrm{E}_{\mathrm{dyn}}$ of the concrete tested in the case of a 1-D analysis. The laser extensometer based on interferometry technique, combined with reflectors made of thin tiles of aluminium alloy glued on the rear face of the specimen, allow to directly obtaining the particles velocity. The signals recorded by the strain gauges and laser extensometers are sometime combined in order to obtain the history of wave propagation along the specimen and hence to determine their velocity $\mathrm{C}_{0}$, and its linearly related Young's modulus $\mathrm{E}_{\mathrm{dyn}}$ of the material tested, and also the failure time [24] .

It should be noted though that these new measuring instruments suffer from some limitations. Thus, the accelerometer glued to rear face of specimen, even of very small mass, may cause considerable local inertial forces when it reaches high accelerations that can locally disrupt the velocity fields or locally damage the specimen during the deceleration stage. As for the laser extensometer, the major disadvantage is that the velocity measurement is punctual (limited to a circular area of about $\sim 20 \mu \mathrm{m}$ diameter), which for an heterogeneous material such as concrete, composed of relatively large aggregates and sand in a cement matrix can represent an important source of uncertainty depending on where the laser is pointing. The same disadvantage with nonetheless less severity characterizes also measures with strain gauges cemented on concrete surface (or embedded in).

Concerning the data processing, spall strengths and the strain rates were deduced in some modified experimental arrangement, as in the original one, by numerical simulation of the waves superposition process in the specimen, knowing the fracture position experimentally determined $[18,20]$. In other experimental setups, strain rates were deduced from direct measurements obtained with strain gauges cemented on specimen surface. It is of course problematic to perform accurate strain measurement, because the sensor device should be located at the exact failure section. Even in this case, it will probably fail at the fracture instant without capturing the complete strain pulse. To overcome this difficulty, strain rates were in this case indirectly estimated from signals measured by the strain gauges located near the failure surface $[23,24]$. The question that arises naturally with this kind of processing is the level of precision of these data knowing that the value of net tensile pulse and the strain rate resulting from superposition process of the transmitted compressive wave can changes considerably along very short travelled distances. This approximation could explain the relative DIF's underprediction at very high strain rate found by Erzar and Forquin [24] when compared to those of other aforementioned authors, Fig. 7. The time deriving of strain-rate from gauge signals in an indirectly estimated position in the damaged area seems rather hazardous.

The need to deal with a representative concrete material to be studied in dynamics in the framework of $1 \mathrm{D}$ analysis of wave propagation explain to some extent the quasi-systematic choice of large dimensions of rods (respectively $240 \mathrm{~mm} \div 1000 \mathrm{~mm}$ and $2000 \mathrm{~mm} \div$ $5250 \mathrm{~mm}$ lengths for the specimen and the Hopkinson bar, for diameters of $45 \mathrm{~mm} \div 75 \mathrm{~mm}$ ). The dynamic compressive loading were generated by means of strikers (60-80 mm length) or detonation generating short lived pulses of $\mathrm{T}=70 \div 100 \mu \mathrm{s}$ duration, and with typical rise and fall times ranging from 20 to $50 \mu \mathrm{s}$. The ratio wavelength-to-specimen diameter takes then a value $\lambda / \phi \sim 1.2$, which let the assumption of a uniaxial propagation of compressive wave with a constant velocity $\mathrm{C}_{0}$ rather questionable. Indeed, it was early established that impulsive loadings, with predominant high frequency components, associated with brittle failures may greatly increase dispersion (attenuation) effects on elastic (viscoelastic) wave propagation in the rods $[7,12,16,17,25,26]$. Because of the geometrical dispersion, the signals measured locally via strain gauges cemented on specimen surface and/or laser extensometer could be strongly distorted and attenuated even in a short traveled distance, and non-uniform along the specimen cross section.

Knowing that the dispersion phenomena have great importance in the data analysis of spalling tests of cylindrical bars, the correction procedures should applied be systematically and the approximation level of the local measurements and/or their extrapolations must also be carefully checked in order to get more precise and consistent results.

\section{Conclusions}

In this paper, a retrospective of the last fifteen years concerning the experimentation for assessing concrete dynamic tensile strength, based on the spalling technique developed in Metz University, highlighted to following main points

- Because its simplicity and efficiency this experimental technique becomes a reference in testing concrete at high strain rates in tension.

- During the last years, this experimentation has known remarkable advances by the introduction of 
modifications like the pulse shaping and the use of detonation charge for a better reproducibility of loading signals, and also data processing based on recorded signals via strain gauges cemented on concrete specimens surfaces and/or contact (or non) measurements (accelerometer, laser extensometer, fast video).

- To deal with a representative concrete material, relatively large specimen diameter were tested, inducing necessarily the adoption of relatively long bars, for analyse on the 1D uniaxial wave framework. However, the geometrical dispersive phenomena induced by finite bars and short dynamic loading with high frequency content, are generally not taken into account in the data processing.

- The data gathered support clearly the considerable and puzzling strength increase of $600 \%$ observed six decades earlier for strain rate greater than $1.0 \mathrm{~s}^{-1}$.

The experimentation is significantly improved by the introduction of direct measurements on concrete loaded specimens. However, the dispersive phenomena must be imperatively taken into account in the data processing. More research efforts are needed to improve the precision of measurements.

\section{References}

[1] L.J. Malvar, C. A. Ross, ACI Mat. J. 95 (6), 735-739 (1998)

[2] Birkimer, D.L., Ph.D. thesis, University of Cincinnati (1968)

[3] Mc Vay M.K., U. S. Army Corps of Engineers, Waterways Experiment Station, Vicksburg, Technical Report SL-88-22 (1998)

[4] Gupta Y. M., Seaman L., Nuclear Eng. and Design 45 (2), 507-514 (1978)

[5] Brara A., Klepaczko J. R.; Kruszka L., in: Proceedings Brittle Matrix Composites 5 (BMC5), Brand, Li, Marshall Eds., Warsaw, Poland (1997), 281-290
[6] Bierwirth S., Najar J., VDI Fortschritt-Berichte ${ }^{\circ}$ 148, reihe 18, 1-148 (1994)

[7] R. M. Davies, Phil. Trans. of the Roy. Soc. of London. Series A, Math. and Phys. Sci. (1948)

[8] Kolsky H. Stress waves in solids, (New York: Dover Publications; 1963)

[9] Pochhammer L., J. Reine Angew. Math., 81, 324-36 (1876)

[10] Chree C., Cambridge Philos. Soc. Trans, 14, 250-369 (1889)

[11] Toutlemonde F., PhD Thesis, Lab. of Public Works (LCPC), Paris, 1994 (in French)

[12] Brara A., Ph.D. thesis, Lab. of Phys. and Mech. of Mat. (LPMM), Metz 1999 (in French)

[13] Klepaczko J. R., Brara A., Int. J. Imp. Engng, 25, 387-409 (2001)

[14] Bailly P., Lab. Netwok GEO, ENI , Bourges final report, Part 1 (1999)

[15] Darve F., Hicher P.Y., Reynouard J-M. Mécanique des geomateriaux, (Hermes Pub., Paris, 1995)

[16] F. Galvez Diaz-Rubio, J. Rodriguez Perez, V. Sanchez Galvez, Int. J. Imp. Engng, 27, 161-177 (2002)

[17] Gomez del Rio T. Rodriguez Perez J., Int. J. Imp. Engng, 34 (2), 377-393 (2007)

[18] Wu H., Zhang O., Huang F., Jinb O., Int. J. Imp. Engng, 32, 605-617 (2005)

[19] Zhidan Rong and Wei Sun, Construction and Building Materials, 31, 168-173 (2012)

[20] Schüler H., Mayrhofer C., Thoma K., Int. J. Imp. Engng, 32, 1635-1650 (2005)

[21] Novikov SA, Divnov II, Ivanov AG Fizika Metallov i Metallovedeniye 21, 4 (1966)

[22] V. Mechtcherine, O. Millon, M. Butler, K. Thoma, Cem. \& Conc.Comp. 33, 1-11 (2011)

[23] Weerheijm J., Van Doormaal J.C.A.M., Int. J. Imp. Engng, 34 (3), 609-626 (2006)

[24] B. Erzar and P. Forquin, Experimental Mechanics 50, 941-955 (2010)

[25] Franz, C., Follansbee, P.S., J. Eng. Mater. Technol. 105, 61-66 (1983) 